Original Article

\title{
Peripheral intravenous catheter practices in Australian and New Zealand neonatal units: A cross-sectional survey
}

\author{
D. August ${ }^{\mathrm{a}, \mathrm{b}, *}$, A.J. Ullman ${ }^{\mathrm{b}}$, C.M. Rickard ${ }^{\mathrm{b}}$, K. New ${ }^{\mathrm{c}}$ \\ a James Cook University, Townsville, Australia \\ ${ }^{\mathrm{b}}$ Alliance for Vascular Access Teaching and Research Group, Menzies Health Institute Queensland and School of Nursing and Midwifery, Griffith University, Brisbane, \\ Australia \\ ${ }^{\mathrm{c}}$ School of Nursing, Midwifery and Social Work, University of Queensland, Brisbane, Australia
}

\section{A R T I C L E I N F O}

\section{Keywords:}

Infusion therapy

Intravenous

Peripheral

Infection prevention

Skin injury

Nursing practice

Neonates

\begin{abstract}
A B S T R A C T
Peripheral intravenous catheters (PIVCs) are important tools for the provision of treatment for unwell and premature neonates. This study aimed to explore current PIVC practices (i.e. insertion, product selection, complications and management priorities) within Australian and New Zealand neonatal units, to identify areas for innovation and practice change. The survey was distributed via Australian and New Zealand neonatal nursing associations, with 180 respondents (54\% Australia; 46\% New Zealand). Respondents reported an average of 2-3 insertion attempts were required per PIVC, with variability in cleansing agents, decontamination techniques, skin barrier films and PIVC dressing products used. The large majority of respondents reported seeing skin complications associated with PIVCs within their practice (94\%). Infection prevention was the highest management priority, and skin complication prevention, the lowest priority. High quality research is necessary to inform neonatal PIVC insertion and management practices, to improve patient safety and treatment provision.
\end{abstract}

\section{Introduction}

Peripheral intravenous catheters (PIVC) are one of the most ubiquitous medical devices, with many neonatal patients requiring their insertion for the administration of medications and/or fluids. Despite being common, PIVC placement can be difficult and time-consuming, particularly for neonatal patients with less visible veins, and compromised skin (Ainsworth and McGuire, 2016). Neonatal skin is thin and structurally immature, increasing the risk of absorption of procedural solutions leading to possible burns, toxicity, or adhesive related injuries (Ponnusamy et al., 2014). The neonatal vascular network also continues to mature after the first year of life (McCullen and Pieper, 2006) and vein structure includes decreased muscle diameter requiring a small gauge cannula (Franck et al., 2001). Clinicians are challenged with site selection for PIVC as particular veins (e.g., cephalic, basilic and brachial veins) are reserved for central venous catheter (CVC) insertion (Beauman and Swanson, 2006).

Evidence for best practice CVC insertion and management significantly outweighs PIVC management (Shah and Tracy, 2013; Sharpe et al., 2016). Recommendations for neonatal PIVC best practice are currently limited to cannula gauge size (Franck et al., 2001), fluids to avoid peripheral infusion (McCullen and Pieper, 2006), and consideration for PIVC complications (Legemaat et al., 2016; Tandale et al., 2017). Restieaux et al. (2013) reported practice variation for extravasation management among Australian and New Zealand Neonatal Units in regards to PIVC and CVC complications. There is a paucity of literature examining the efficacy of many neonatal PIVC insertion and management practices, including skin decontamination products, catheter security and dressing selection within neonates. In the absence of evidence for best practice for neonatal PIVCs, neonates are likely to suffer additional discomfort and distress, interruptions to treatment, increased risk of infection, and venous depletion in later life.

Practice surveys can be used to identify gaps in clinical practices and knowledge (Shah and Tracy, 2013), highlighting future directions for research and practice development. This study was undertaken to guide future direction of research by this research team. This study aimed to describe PIVC practices within Australian and New Zealand neonatal units including (a) PIVC insertion, (b) decontamination and dressing product selection, (c) frequency of PIVC-associated skin complications, and (d) PIVC management priorities.

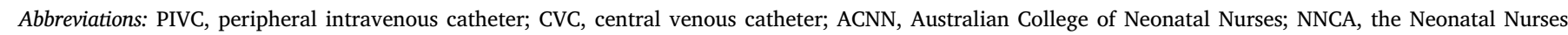
College Aotearoa; CHG, chlorhexidine gluconate

* Corresponding author. Brisbane, QLD 4006, Australia.

E-mail address: d.august@griffith.edu.au (D. August). 


\section{Methods}

\subsection{Design}

A cross-sectional, descriptive survey of neonatal PIVC practices was conducted via the Australian College of Neonatal Nurses (ACNN) and the Neonatal Nurses College Aotearoa (NNCA; New Zealand) between August and October 2016. Ethics approval was gained through Griffith University, prior to study commencement (NRS/2016/698).

\subsection{Data collection and instruments}

This survey was developed in accordance with previous audits and surveys for vascular access devices, with content specifically altered for relevance in the neonatal setting (Alexandrou et al., 2015; Ullman et al., 2017). The survey requested the respondents to describe their actual practice and priorities, rather than local protocols or guidelines for PIVC practices. The survey included 15 questions, surrounding: (a) PIVC insertion (i.e. average number of PIVC attempts required, clinicians inserting); (b) decontamination and dressing product selection (c) frequency of PIVC associated skin complications (i.e. skin reactions [such as dermatitis or redness] (McNichol et al., 2013) and skin injuries [i.e. adhesive and pressure injuries] (August et al., 2017; Broadhurst et al., 2017)) across a frequency-based 5-point Likert scale [1 = Never; $5=$ Always]) and (d) PIVC management priorities using a three point prioritisation scale across the categories of infection prevention, PIVC function and skin health. Basic demographic and professional data were collected to describe the respondents (country of residence, years of neonatal practice and neonatal unit classification). Neonatal unit classifications were described in accordance with the Queensland Neonatal Services Levels of Care (Department of Health, 2016).

Survey participation was voluntary and anonymous. The survey was distributed via two methods. Paper copies were distributed to attendees of the ACNN National Conference (187 attendees; 22-24th September 2016; Melbourne, Australia) and NNCA National Conference (99 attendees: $26^{\text {th }}-28$ th October 2016; Dunedin, New Zealand). Following the conferences, a link to an electronic survey, via LimeSurvey ${ }^{\circ}$, was emailed to ACNN $(n=682)$ and NNCA members $(n=600)$. Respondents were instructed to complete the survey only once and only if they had not completed a paper copy at a conference. Responses received via paper copy were entered manually into LimeSurvey ${ }^{\circ}$.

\subsection{Data analysis}

Standard data cleaning and checking procedures were undertaken prior to analysis. Results are reported per survey respondent, with a single answer per respondent unless otherwise explained. Descriptive statistics including frequency and percentages for categorical variables; and mean, standard deviations (SD) and ranges for continuous variables were used. Data were analysed using PASW 22.0 (SPSS Inc, Chicago, IL, USA). Missing data are described throughout the results tables.

\section{Results}

\subsection{Respondent demographics}

Surveys were completed by approximately $14 \%$ of clinicians with membership to ACNN or NCAA ( $=180$ ), with $54 \%$ from Australia $(\mathrm{n}=97)$ and $46 \%$ from New Zealand $(\mathrm{n}=83$ ). Respondent demographics are reported in Table 1 . The majority of respondents $(n=145$, $81 \%$ ) had greater than seven years' neonatal experience. Respondents predominately worked in Level 6 or equivalent units (i.e. the highest level of care) ( $n=107,59 \%)$.

Only $16 \%(n=29)$ of respondents inserted PIVCs frequently with the majority ( $n=60,60 \%$ ) not inserting PIVCs at all. The majority of respondents ( $\mathrm{n}=160,89 \%)$ reported that, in their clinical experience, the average number of skin punctures or attempts to insert a neonatal PIVC was greater than two.

\subsection{PIVC decontamination and product selection}

As displayed in Table 2, there were substantial variations in cleansing agent type, preparation, application and removal associated with PIVCs. While most respondents reported using a chlorhexidine gluconate (CHG)-based product for site decontamination prior to PIVC insertion ( $n=150,83 \%)$, the CHG concentration varied from 0.01 to $2 \%$. CHG formulation varied including aqueous suspensions or combined antiseptic solutions (CHG in alcohol or Cetrimide), and antisepsis preparation (solution, pre-packaged swab, swab stick). Agent preparations primarily used were single use or pre-packaged $(n=152,84 \%)$. Finally, some respondents $(n=31,18 \%)$ reported removal of the cleansing agent (e.g., CHG) with either sterile gauze and/or water prior to PIVC insertion.

Data surrounding PIVC dressing and securement are described in Table 3. At least 11 primary and secondary dressing products were reported. Polyurethane films were the most common product used to secure PIVCs $(\mathrm{n}=158,88 \%)$, with additional securement provided by a variety of adhesives including steri-strips $(\mathrm{n}=97,54 \%)$, non-sterile fabric $(n=59,33 \%)$, paper $(n=6,3 \%)$ and acrylic tapes $(n=5,3 \%)$. About one third of the respondents $(n=57,32 \%)$ suggested splints were utilised, although type and size were not ascertained.

\subsection{Skin complications}

On average, respondents reported skin reactions and skin injuries occurred rarely or sometimes at the PIVC site (mean 2.5; SD 0.6-7) (Table 4). Comparatively, skin injuries were observed marginally more often $(n=147,97 \%)$ compared to skin reactions $(n=168,93 \%)$. Only a small percentage $(<6 \%)$ had never seen a skin complication at a PIVC site.

\subsection{PIVC insertion and management priorities}

Respondents described mixed priorities when deciding between PIVC insertion and securement practices (see Table 5). While $50 \%$ of respondents $(n=71)$ prioritised infection prevention, $41 \%$ prioritised PIVC function and accessibility $(n=58)$. The prevention of skin complication was the first priority for $6 \%$ of respondents $(n=8)$, however $22 \%(n=39)$ reported each goal to be equally important.

Table 1

Respondents demographics and role in PIVC insertion $(n=180)$.

\begin{tabular}{llll}
\hline & & $\mathrm{n}$ & $\%$ \\
\hline \multirow{2}{*}{ Country } & Australia & 97 & 54 \\
Neonatal unit classification & New Zealand & 83 & 46 \\
& Level 6 & 107 & 59 \\
& Level 3-5 & 69 & 38 \\
& Level 1-2 & 4 & 2.2 \\
Years practice in neonates & $<1$ year & 0 & 0 \\
& $1-3$ years & 10 & 6 \\
& $>3-7$ years & 23 & 13 \\
& $>7$ years & 145 & 81 \\
Role in PIVC insertion & Missing & 2 & 1.1 \\
& Commonly insert & 29 & 16 \\
& Occasionally insert & 41 & 23 \\
& No - but would like to & 55 & 31 \\
& No - I do not wish to & 52 & 29 \\
& Missing & 3 & 1.8 \\
\hline
\end{tabular}

*multiple responses per respondent; PIVC = Peripheral intravenous catheter. 
Table 2

Preparation, application, removal of decontamination agents $(n=180)$.

\begin{tabular}{|c|c|c|c|}
\hline & & $\mathrm{N}$ & $\%$ \\
\hline \multirow[t]{8}{*}{ Decontamination agent used at the insertion site before placing a PIVC } & $2 \%$ CHG in $70 \%$ alcohol & 50 & 28 \\
\hline & $2 \%$ Aqueous $\mathrm{CHG}$ & 47 & 26 \\
\hline & $0.5 \%$ CHG in $70 \%$ alcohol & 38 & 21 \\
\hline & Alcohol & 13 & 7 \\
\hline & Other CHG-based products & 11 & 6.1 \\
\hline & $0.01 \%$ CHG with Cetrimide & 4 & 2.2 \\
\hline & Povidone Iodine & 2 & 1.1 \\
\hline & Other & 15 & 8 \\
\hline \multirow[t]{5}{*}{ Decontamination agent application type } & Single use solution & 63 & 35 \\
\hline & Pre-packaged swab & 59 & 33 \\
\hline & Pre-packaged swab stick & 30 & 17 \\
\hline & Multiple use solution & 24 & 13 \\
\hline & Other & 4 & 2 \\
\hline \multirow[t]{4}{*}{ Decontamination agent application method } & Circulation motion, inside outwards & 114 & 63 \\
\hline & Back and forth & 55 & 31 \\
\hline & Dabbing & 3 & 2 \\
\hline & Other & 8 & 4 \\
\hline \multirow[t]{5}{*}{ Decontamination removal agent used prior to PIVC insertion } & None & 143 & 79 \\
\hline & Wiped with sterile gauze & 27 & 15 \\
\hline & Wiped with sterile water & 4 & 2.3 \\
\hline & Other & 3 & 1.8 \\
\hline & Missing & 3 & 1.8 \\
\hline
\end{tabular}

CHG $=$ Chlorhexidine gluconate; PIVC $=$ Peripheral intravenous catheter.

Table 3

Barrier films, dressings, and securements for PIVCs.

\begin{tabular}{|c|c|c|c|}
\hline & & $\mathrm{N}$ & Percentage \\
\hline \multirow[t]{4}{*}{ Barrier film use prior to dressing application $(\mathrm{n}=180)$} & None & 161 & 89 \\
\hline & Cavilon (3M) & 5 & 3 \\
\hline & Skin Prep (Smith \& Nephew) & 3 & 2 \\
\hline & Other & 11 & 6 \\
\hline \multirow[t]{6}{*}{ Primary dressings* } & Steri-strips & 97 & 54 \\
\hline & Polyurethane film with border & 92 & 51 \\
\hline & Polyurethane film (no border) & 66 & 37 \\
\hline & Non-sterile fabric tape & 59 & 33 \\
\hline & Paper tapes & 6 & 3 \\
\hline & Non-sterile acrylics (plastic tapes) & 5 & 3 \\
\hline \multirow[t]{9}{*}{ Secondary dressings and securements* } & Arm boards & 121 & 67 \\
\hline & Fabric tape & 105 & 58 \\
\hline & Splints & 57 & 32 \\
\hline & Polyurethane film & 24 & 13 \\
\hline & Foam tape & 12 & 7 \\
\hline & Non-sterile tapes (hyperfix) & 10 & 5 \\
\hline & Securement device & 4 & 2 \\
\hline & Other & 5 & 3 \\
\hline & None & 3 & 2 \\
\hline
\end{tabular}

*multiple responses per respondent; PIVCs = Peripheral intravenous catheters.

\section{Discussion}

This is the first survey to describe current PIVC practices within Australian and New Zealand neonatal units. Despite low response rates, this survey demonstrated many areas of neonatal PIVC practice variability, and opportunities for improvement. While neonatal PIVCs may appear to present less risk than CVCs; PIVCs are increasingly associated with complications such as infection and skin injury (August et al., 2014; Liversedge et al., 2018; Nist et al., 2016; Sweeney et al., 2018). With an understanding of current practice variability and its sequelae, innovation in PIVC science is warranted (Pettit, 2003).

Respondents reported PIVC insertion in neonates as challenging, with an average of two or more attempts required, with PIVCs primarily inserted by physicians in this study. Similar challenges were identified in a study during which PIVCs were mainly inserted by nurses (mean PIVC attempts $2.2 \pm 1.67$; range $1-12, \mathrm{n}=207$ ) suggesting inserter discipline may not factor into these difficulties (Franck et al., 2001). More recently in the Netherlands, Legemaat et al. (2016) also found
Table 4

Skin complications associated with PIVC $(n=180)$.

\begin{tabular}{clll}
\hline & & $\mathrm{n}$ & Percentage \\
\hline Skin reactions observed at PIVC site (such as & Never & 10 & 6 \\
dermatitis or redness) & Rarely* $^{*}$ & 81 & 45 \\
& Sometimes* & 70 & 39 \\
& Frequently & 17 & 9 \\
& Always & 0 & 0 \\
& Missing & 2 & 1.1 \\
\cline { 2 - 4 } Skin injuries observed at PIVC site (such as & Never & 4 & 2 \\
adhesive or pressure injuries) & Rarely** & 87 & 48 \\
& Sometimes** & 77 & 43 \\
& Frequently & 10 & 6 \\
& Always & 0 & 0 \\
& Missing & 2 & 1.1 \\
\hline
\end{tabular}

PIVC $=$ Peripheral intravenous catheter; $\mathrm{SD}=$ Standard deviation.

*Mean (SD; range) $2.5(0.7 ; 1-4)$

**Mean (SD; range) $2.5(0.6 ; 1-4)$. 
Table 5

Priorities for PIVC insertion and management practices $(n=180)$.

\begin{tabular}{|c|c|c|c|}
\hline & 1st Priority (n,\%) & 2nd Priority (n,\%) & 3rd Priority $(n, \%)$ \\
\hline Infection prevention & $71(50)$ & $43(30)$ & $17(12)$ \\
\hline Function and access & $58(41)$ & $52(37)$ & $26(18)$ \\
\hline Skin health (Prevention of skin complications) & $8(6)$ & $38(27)$ & $87(62)$ \\
\hline Equally important (All three priorities) & $39(22)$ & - & - \\
\hline Other (Only 2 of 3 priorities selected) & $3(2)$ & $1(1)$ & 0 \\
\hline Missing & $1(0.5)$ & $7(3.8)$ & $11(6.1)$ \\
\hline
\end{tabular}

PIVC $=$ Peripheral intravenous catheter.

first insertion success rates to be only 234/518 (45\%); lower than in general populations. The Infusion Therapy Standards of Practice (Infusion Nurses Society, 2016) advocate for a maximum of two attempts per clinician for any patient population. However, the limited number of studies in the neonatal population indicates that at least two attempts are required for successful peripheral cannulation. Given the fragility of this population and their vascular structure further research is required regarding the effect of clinician experience on successful cannulation. This will inform recommendations as to the maximum number of cannulation attempts by a single clinician, to avoid resultant damage to veins that are needed over the course of a lifetime.

There was variation in reported decontamination practices, including product selection, application and removal. Most decontamination agents reported contained a CHG based product, comparable with 60-90\% CHG use in previous studies (Tamma et al., 2010; Shah and Tracy, 2013). The use of CHG products for patients less than 2-3 months of age remains controversial due to a high rate of skin complications (Ponnusamy et al., 2014). Respondent answers reflected that the method of application for decontamination products was primarily as per clinical practice guidelines (Infusion Nurses Society, 2016; O'Grady et al., 2011) using a circular motion, with inward to outward direction $(n=114,63 \%)$. As $18 \%$ of respondents reported the subsequent removal of the decontamination agent, education is likely needed in relation to leaving agents to dry as has also been demonstrated in recent studies (Pettit and Sharpe, 2017).

While polyurethane/transparent films were slightly favoured for the securement of PIVCs, a variety of adhesive products were selected by respondents. Non-sterile, paper and acrylic tapes are still being used in practice for primary securement of PIVCs. These particular adhesives are associated with higher rates of skin injuries (Boswell and Waker, 2016; Lund, 2014) and potential risk for infection (Infusion Nurses Society, 2016). Tapes may also decrease the speed of assessment for extravasation complications, compared to transparent dressings (McCullen and Pieper, 2006). A consistent and effective approach to PIVC dressing and security is necessary to prevent patient harm (Marsh et al., 2015).

Respondents reported that skin barrier film products are infrequently used in Australian and New Zealand neonatal practice. This may reflect the inconclusive evidence for barrier product safety and efficacy in the neonatal population. Most trials evaluating skin barrier films have focused on other population groups (e.g., the elderly) and/or clinical indications (e.g., chronic wounds) (Schuren et al., 2005). Additionally, few of the products available advocate safety in neonates younger than 30 days (Brandon et al., 2010). Further clinical trials evaluating the effectiveness of skin barrier films to prevent skin complications surrounding vascular access devices are necessary.

The respondents reported that in their clinical practice, skin complications surrounding PIVC sites occurred rarely or sometimes. This is in contrast to studies which highlight that vascular access devices are among the most common device related to neonatal pressure and adhesive injuries (August et al., 2017; Habiballah, 2017; Liversedge et al., 2018; Nist et al., 2016; Sweeney et al., 2018). It may also reflect a lack of understanding of skin injuries and reactions that seem innocuous such as redness and adhesive related injuries. Understanding the causes and strategies to prevent hospital acquired complications such as skin injuries will require further research. While this study did not report on them, additional complications should also be considered including PIVC infiltration, extravasation, leakage, occlusion and the related infusates.

The majority of respondents ranked infection prevention as the top priority for the decontamination; securement and management of PIVCs, the promotion of PIVC functionality second; and the prevention of PIVC-associated skin complications as the lowest priority. However, many $(22 \%, \mathrm{n}=39)$ respondents ranked infection prevention, PIVC functionality, and skin complications of equal importance, highlighting the need for PIVC practices that promote all three priorities. While PIVC-associated infections in neonatal units are rare, the sequelae is devastating (Leibovitz et al., 1992), with bloodstream infections associated with considerable morbidity and mortality (Ponnusamy et al., 2014). Previous literature has also highlighted the frequency of PIVC failure within neonatal units due to infiltration, accidental dislodgement and phlebitis, causing treatment delays and discomfort (Legemaat et al., 2016).

\subsection{Limitations}

The low response rate for this survey limits generalisability to some extent, and this risk self-selection bias is typical for research conducted with online distribution or at conferences. In addition, responses were based on reflections on practice, rather than direct observation, therefore may not correctly reflect actual clinical practice. Respondents may have given answers they believed as socially desirable rather than their actual practice. Finally, these results represent the practices of Australia and New Zealand neonatal units and may not reflect other regions.

\section{Conclusion}

PIVC insertion and management practices in neonatal units vary considerably across Australia and New Zealand. Further research is needed on neonatal PIVC insertion and management to establish the efficacy of decontamination agents, dressing and securement products, and skin barrier films, to prevent patient harm and promote treatment administration.

\section{Conflicts of interest}

Portions of these results have been presented at the Australian College of Neonatal Nurses (2017) National Conference and the Association for Vascular Access Annual Scientific Meeting. Griffith University has received unrestricted, and investigator-initiated funding from vascular access product manufacturers (e.g., 3M, Angiodynamics, Becton Dickinson, Centurion Medical Products), unrelated to this project, to support the research of AJU and CRM.

\section{Funding}

No funding has been received to undertake this research. 


\section{References}

Ainsworth, S.B., McGuire, W., 2016. Peripherally inserted central catheters vs peripheral cannulas for delivering parenteral nutrition in neonates. J. Am. Med. Assoc. 315, 2612-2613.

Alexandrou, E., Ray-Barruel, G., Carr, P.J., Frost, S.A., Inwood, S., Higgins, N., Lin, F. Alberto, L., Mermel, L., Rickard, C., 2015. International prevalence of the use of peripheral intravenous catheters. J. Hosp. Med. 10, 530-533.

August, D.L., Edmonds, L., Brown, D.K., Murphy, M., Kandasamy, Y., 2014. Pressure injuries to the skin in a neonatal unit: fact or fiction. J. Neonatal Nurs. 20, 129-137.

August, D.L., New, K., Ray, R., Kandasamy, Y., 2017. Frequency, location and risk factors of neonatal skin injuries from mechanical forces of pressure, friction, shear and stripping: a systematic literature review. J. Neonatal Nurs. 24, 173-180.

Beauman, S., Swanson, A., 2006. Neonatal infusion therapy: preventing complications and improving outcomes. N.born Infant Nurs. Rev. 6, 193-201.

Boswell, N., Waker, C.L., 2016. Comparing 2 adhesive methods on skin integrity in the high-risk neonate. Adv. Neonatal Care 16, 449-454.

Brandon, D., Coe, K., Hudson-Barr, D., Oliver, T., Landerman, L., 2010. Effectiveness of No-Sting skin protectant and Aquaphor on water loss and skin integrity in premature infants. J. Perinatol. 30, 414-419.

Broadhurst, D., Moureau, N., Ullman, A.J., 2017 May/Jun. Management of central venous access device-associated skin impairment: an evidence-based algorithm. J Wound Ostomy Cont 44 (3), 211-220. https://doi.org/10.1097/WON.0000000000000322.

Department of Health, 2016. In: Health, D.o. (Ed.), Neonatal Services, Clinical Services Capability Framework. Department of Health, Queensland Government, Brisbane, Queensland, Australia.

Franck, L., Hummel, D., Connell, K., Quinn, D., Montgomery, J., 2001. The safety and efficacy of peripheral intravenous catheters in ill neonates. Neonatal Netw. 20, 33-38.

Habiballah, L., 2017. Prevalence of neonate adhesive skin injuries in a Jordanian intensive care unit. Nurs. Child. Young People 29, 42-46.

Infusion Nurses Society, 2016. Infusion therapy standards of practice. J. Infusion Nurs. 39.

Legemaat, M., Carr, P., van Rens, R., van Dijk, M., Poslawsky, I., van den Hoogen, A., 2016. Peripheral intravenous cannulation: complication rates in the neonatal population: a multicenter observational study. J. Vasc. Access 17, 360-365.

Leibovitz, E., Iuster-Reicher, A., Amitai, M., Mogilner, B., 1992. Systemic candidal infections associated with use of peripheral venous catheters in neonates: a 9-year experience. Clin. Infect. Dis. 14 485-49.

Liversedge, H.L., Bader, D.L., Schoonhoven, L., Worsley, P.R., 2018. Survey of neonata nurses' practices and beliefs in relation to skin health. J. Neonatal Nurs. 24, 86-93. Lund, C., 2014. Medical adhesives in the NICU. N.born Infant Nurs. Rev. 14, 160-165.

Marsh, N., Webster, J., Mihala, G., Rickard, C.M., 2015. Devices and dressings to secure peripheral venous catheters to prevent complications. In: Cochrane Database Syst
Rev, pp. Cd011070.

McCullen, K.L., Pieper, B., 2006. A retrospective chart review of risk factors for extravasation among neonates receiving peripheral intravascular fluids. J Wound Ostomy Cont 33, 133-139.

McNichol, L., Lund, C., Rosen, T., Gray, M., 2013. Medical adhesives and patient safety: state of the science: consensus statements for the assessment, prevention, and treatment of adhesive-related skin injuries. Orthop. Nurs. 32, 267-281.

Nist, M.D., Rodgers, E.A., Ruth, B.M., Bertoni, C.B., Bartman, T., Keller, L.A., Dail, J.W., Gardikes-Gingery, R., Shepherd, E.G., 2016. Skin Rounds: a quality improvement approach to enhance skin care in the neonatal intensive care unit. Adv. Neonatal Care 16 (Suppl. 5S), S33-S41.

O'Grady, N.P., Alexander, M., Burns, L.A., Dellinger, E.P., Garland, J., O'Heard, S., Lipsett, P.A., Masur, H., Mermel, L.A., Pearson, M.L., Raad, I.I., Randolph, A., Rupp, M.E., Saint, S., HICPAC, H.I.C.P.A.C., 2011. Guidelines for the prevention of intravascular catheter-related infections. Clin. Infect. Dis. 52, e162-193.

Pettit, J., 2003. Assessment of the infant with a peripheral intravenous device. Adv. Neonatal Care 3, 230-240.

Pettit, J.D., Sharpe, E.L., 2017. The effect of education on chlorhexidine use in a neonatal intensive care unit. J. Vasc. Access 22, 115-123.

Ponnusamy, V., Venkatesh, V., Clarke, P., 2014. Skin antisepsis in the neonate: what should we use? Curr. Opin. Infect. Dis. 27, 244-250.

Restieaux, M., Maw, A., Broadbent, R., Jackson, P., Barker, D., Wheeler, B., 2013. Neonatal extravasation injury: prevention and management in Australia and New Zealand-a survey of current practice. BMC Pediatr. 13, 34.

Schuren, J., Becker, A., Sibbald, R.G., 2005. A liquid film-forming acrylate for peri-wound protection: a systematic review and meta-analysis (3M Cavilon no-sting barrier film). Int. Wound J. 2, 230-238.

Shah, D., Tracy, M., 2013. Skin antisepsis survey in Australia-New Zealand neonatal nurseries. Int. J. Pediatr. Child Health 49, 601-602.

Sharpe, E., Kuhn, L., Ratz, D., Krein, S.L., Chopra, V., 2016. Neonatal peripherally inserted central catheter practices and providers: results from the neonatal PICC1 survey. Adv. Neonatal Care 17, 209-221.

Sweeney, K.A., Cogill, K., Davis, K., Jauncey-Cooke, J.I., 2018. Neonatal and pediatric pressure injuries secondary to limb splinting for intravascular access: case series and literature review. J. Vasc. Access 23, 108-116.

Tamma, P.D., Aucott, S.W., Milstone, A.M., 2010. Chlorhexidine use in the neonatal intensive care unit: results from a national survey. Infect. Control Hosp. Epidemiol. 31, $846-849$.

Tandale, S., Dave, N., Garasia, M., Patil, S., Parelkar, S., 2017. A study of morbidity and cost of peripheral venous cannulation in neonates admitted to paediatric surgical intensive care unit. J. Clin. Diagn. Res. 11, UC08-10.

Ullman, A.J., Cooke, M., Kleidon, T., Rickard, C.M., 2017. Road map for improvement: point prevalence audit and survey of central venous access devices in paediatric acute care. J. Paediatr. Child Health 53, 123-130. 